\title{
Die Kontrolle der Tuberkulose in den Nachfolgestaaten der Sowjetunion am Beispiel Russlands
}

\section{Control of Tuberculosis in Russia and Other Countries of the Former Soviet Union}

Autoren

Institute
F. M. Marx ${ }^{1}$, E. I. Skachkova², I. M. Son², A. K. Strelis ${ }^{3}$, O. I. Urazova ${ }^{3}$, H. Hahn ${ }^{1}$, A. Krämer ${ }^{4}$, T. Ulrichs $s^{1,4}$

Sektion Tuberkulose, Koch-Metschnikow-Forum, Berlin

Federal Tuberculosis Monitoring Centre, Public Health Institute, Moskau, Russische Föderation

Sibirische Staatliche Medizinische Universität Tomsk, Russische Föderation

${ }^{4}$ Fakultät für Gesundheitswissenschaften, Universität Bielefeld eingereicht 15.10 .2008 akzeptiert nach Revision 9.1.2009

\section{Bibliografie}

DOI $10.1055 / \mathrm{s}-0028-1119571$

Online-Publikation: 3. 3. 2009

Pneumologie 2009; 63: 253-260

(c) Georg Thieme Verlag KG

Stuttgart $\cdot$ New York

ISSN 0934-8387

Korrespondenzadresse

\section{Dr. Timo Ulrichs}

Koch-Metschnikow-Forum

Luisenstr. 59

10117 Berlin

timo.ulrichs@bmg.bund.de

\section{Zusammenfassung \\ $\nabla$}

Hintergrund: Die Tuberkulose bleibt ein ernst zu nehmendes Problem in den Nachfolgestaaten der ehemaligen Sowjetunion. Der Artikel beschreibt die gegenwärtige Situation der Tuberkuloseepidemie in Russland sowie die Besonderheiten der Tuberkulosekontrolle, die sich aus dem traditionellen sowjetischen Kontrollmodell ergeben. Aktuelle Herausforderungen der Tuberkulosekontrolle werden diskutiert.

Methodik: Es wurden Meldedaten der Weltgesundheitsorganisation (WHO) zur Tuberkulose in Russland und 14 weiteren Sowjet-Nachfolgestaaten ausgewertet. Im Rahmen einer Medline/Embase-basierten Analyse wurden 66 Original- und Übersichtsarbeiten zur Tuberkulosekontrolle in Russland untersucht. Zusätzlich wurden russische Berichte und Leitlinien ausgewertet.

Ergebnisse: Im Jahr 2006 wurden in Russland ca. 125000 Tuberkulosefälle und 28000 Sterbefälle registriert. Die Tuberkuloserate war 13-mal so hoch wie in Deutschland. Zu den Charakteristika des traditionellen Kontrollmodells zählen i) die Organisation der Diagnostik und Behandlung im Rahmen eines zentralisierten, krankheitsspezifischen Netzwerks; ii) landesweite Bevölkerungsscreenings mittels Fluorographie; iii) der Schwerpunkt auf Röntgenbefunden für die Diagnosestellung; iv) individualisierte und langfristige stationäre Behandlungen; v) hohe Medikamentenresistenzraten sowie vi) ineffiziente Finanzierungssysteme. Schlussfolgerungen: Wichtigste Herausforderungen der Tuberkulosekontrolle in Russland sind derzeit der Ausbau einer qualitätsgesicherten mikrobiologischen Diagnostik und Resistenztestung; die Behandlung und Kontrolle der multiresistenten Tuberkulose; Prävention und Management von Tuberkulose/HIV und die Reform der Finanzierungssysteme. Dabei muss den Charakteristika des traditionellen Modells der Tuberkulosekontrolle Rechnung getragen werden.

\section{Abstract \\ $\nabla$}

Background: Tuberculosis (TB) remains a serious threat to public health in Russia and other former Soviet Union Countries. The purpose of this paper is to describe the current trends of TB and MDR$\mathrm{TB}$ in Russia and identify the characteristics of the traditional Russian TB control model inherited from the Soviet Union. We discuss current challenges to TB control in the country.

Methods: WHO tuberculosis notification data were analysed for Russia and 14 other former Soviet Union countries. To investigate the characteristics of TB control in Russia, we performed a systematic literature review using MEDLINE/EMBASE databases. 136 articles were initially identified of which 66 fulfilled the inclusion criteria. Full texts were reviewed. Additionally, we reviewed non-systematically Russian state reports, guidelines and legislations.

Results: In 2006, nearly 125000 TB cases and 28000 TB deaths were notified in the Russian Federation. The TB notification rate was 13 times higher than in Germany. The characteristics of the traditional Russian TB control model include: a centralised disease-specific inpatient network for diagnosis and treatment of TB, countrywide population screenings using fluorography, a strong focus on X-ray for diagnosis and disease classification, individualised and lengthy inpatient care, high rates of drug resistance, and inefficient financing systems.

Conclusions: Current challenges to TB control in Russia are: the implementation of a quality-assured laboratory network for sputum-smear microscopy, culture and drug susceptibility testing, ensuring MDR-TB treatment and control, prevention and management of TB/HIV, and reform of health care financing systems. For TB control to be successful in the Russian Federation, the characteristics of the traditional TB control model need to be taken into account. 


\section{Einleitung \\ $\nabla$}

Die Kontrolle der Tuberkulose in Russland und den übrigen Nachfolgestaaten der ehemaligen Sowjetunion steht gegenwärtig vor besonderen Herausforderungen.

Seit Anfang der 90er-Jahre stieg die Zahl neuer Tuberkulosefälle in diesen Ländern von ca. 97000 (1991) auf rund 250000 Fälle um die Jahrtausendwende und verbleibt seitdem auf hohem Niveau. Zunehmende Raten resistenter und multiresistenter Tuberkulosefälle verkomplizieren die Situation.

In den 90er-Jahren wurde auch in den Ländern der ehemaligen Sowjetunion damit begonnen, das von der Weltgesundheitsorganisation (WHO) entwickelte Modell zur Tuberkulosekontrolle, „DOTS“ (directly observed therapy, short course) [1] einzuführen. Die internationale Strategie trifft dabei auf existierende Gesundheits- und Kontrollsysteme, welche ihre Wurzeln im sowjetischen System haben und seither kaum Reformen unterzogen wurden. Bei der Etablierung internationaler Standards ergeben sich daher spezielle Probleme und Herausforderungen, die im Sinne einer erfolgreichen Tuberkulosekontrolle berücksichtigt werden müssen.

Russland, das größte der Sowjet-Nachfolgeländer, liegt nach Angaben der WHO gegenwärtig auf Rang 11 der 22 so genannten High Burden Countries weltweit (nach geschätzter Zahl neuer Tuberkulosefälle 2006, vgl. [2]). Seine geopolitische Bedeutung und die Nähe zur Europäischen Union erwecken auch hinsichtlich der Bekämpfung von Infektionskrankheiten zunehmend die Aufmerksamkeit der internationalen Gemeinschaft. So wurde die Bekämpfung der Infektionskrankheiten und insbesondere der Tuberkulose auf dem G8-Gipfel in St. Petersburg als wichtiges Ziel der Politik der G8-Staaten festgeschrieben.

Der Artikel beschreibt am Beispiel Russlands die gegenwärtige Tuberkuloseepidemie und untersucht die Besonderheiten der Tuberkulosekontrolle in den Nachfolgestaaten der ehemaligen Sowjetunion. Ausgehend von diesen Besonderheiten werden aktuelle Probleme und Herausforderungen der Tuberkulosekontrolle in Russland diskutiert.

\section{Methodik}

$\nabla$

Es wurden Meldedaten der Weltgesundheitsorganisation (WHO) zur Epidemiologie der Tuberkulose für Russland im Vergleich zu 14 weiteren Nachfolgestaaten der ehemaligen Sowjetunion für den Zeitraum 1985-2006 ausgewertet.

Um die Besonderheiten der Tuberkulosekontrolle in Russland zu untersuchen, führten wir eine Analyse der englischsprachigen Literatur durch. In die Studie eingeschlossen wurden Studien mit für Russland spezifischen Informationen über 1.) die Epidemiologie der Tuberkulose im Land; 2.) Aspekte der Tuberkulosekontrolle und Surveillance und 3.) Programme zur Behandlung und Kontrolle der Tuberkulose und multiresistenten (multi drug resistant; MDR-) Tuberkulose.

Für die Literatursuche wurden die Fachdatenbanken MEDLINE und EMBASE verwendet. Folgende Kombination von Schlüsselbegriffen wurde gewählt: (tuberc* OR tb OR mdr* OR phthis*) AND (Russia* OR Soviet*) AND (control OR surveillance OR dots OR program*). Als weitere Suchkriterien wurden verwendet: Original- und Übersichtsartikel (peer-reviewed), publiziert im Zeitraum 1992 - 2008, in englischer und deutscher Sprache. Die Literatursuche ergab initial 136 Treffer. Die Abstracts dieser Artikel wurden in Bezug auf die Einschlusskriterien (s.o.) untersucht. 66
Tab. 1 Eckdaten der Tuberkulose in Russland und Deutschland (2006); Quelle: WHO.

\begin{tabular}{|c|c|c|}
\hline Tuberkulose 2006 & Russland & Deutschland \\
\hline Einwohner & 143221000 & 82641000 \\
\hline \multicolumn{3}{|l|}{ Gemeldete Tuberkulosefälle } \\
\hline - absolut & 124689 & 5402 \\
\hline - Rate pro 100000 Einwohner & 87,0 & 6,6 \\
\hline \multicolumn{3}{|l|}{$\begin{array}{l}\text { Neue pulmonale Tuberkulose- } \\
\text { fälle }\end{array}$} \\
\hline - absolut & 105587 & 3840 \\
\hline - Anteil laborbestätigter Fälle (\%) & 44,0 & 77,0 \\
\hline \multicolumn{3}{|l|}{$\begin{array}{l}\text { Multiresistente (MDR-) } \\
\text { Tuberkulose }\end{array}$} \\
\hline - Resistenztests (\% gemeldeter & $30200(24,2)$ & $3501(64,8)$ \\
\hline Tuberkulosefälle) & $3949(13,1)$ & $78(2,6)$ \\
\hline \multicolumn{3}{|l|}{ - MDR-Fälle (\% getesteter Fälle) } \\
\hline \multicolumn{3}{|l|}{ Tuberkulose und HIV } \\
\hline $\begin{array}{l}\text { - HIV Tuberkulosefälle } \\
\text { (\% getesteter Fälle) }\end{array}$ & $1979(2,2)$ & - \\
\hline \multicolumn{3}{|l|}{ Tuberkulosemortalität } \\
\hline - absolut (Sterbefälle) & 27835 & 201 \\
\hline - Rate pro 100000 Einwohner & 19,5 & 0,2 \\
\hline
\end{tabular}

Artikel erfüllten die Kriterien und wurden in die Studie eingeschlossen. Ihre Volltexte wurden ausgewertet.

Zusätzlich wurden nicht-systematisch Berichte, Verordnungen und Leitlinien des Gesundheitsministeriums der Russischen Föderation zu den Themen Behandlung, Monitoring und Kontrolle, Bevölkerungsscreening und Prävention der Tuberkulose sowie Tuberkuloseberichte der WHO ausgewertet. Ergänzend wurden im Jahr 2007 Experteninterviews mit verantwortlichen Akteuren der Tuberkulosegesundheitsdienste einer Pilotregion der internationalen Strategie (Tomsk Oblast) und einer Region mit Kontrollstrategie nach traditionellem russischen Modell (Republik Tuva) durchgeführt.

\section{Ergebnisse}

\section{Epidemiologie der Tuberkulose in Russland}

Im Jahr 2006 wurden in der Russischen Föderation knapp 125000 Tuberkulosefälle (neue Fälle und Rezidive) sowie 28000 Sterbefälle registriert. Die Rate gemeldeter Fälle pro 100000 Einwohner war 13,2-mal so hoch wie in Deutschland (vgl. $\bullet$ Tab. 1). Nach 2,8-fachem Anstieg der Tuberkuloserate im Zeitraum 1991 bis 2000 (von 34 auf 95 Fälle pro 100000 Einwohner) haben sich die Fallzahlen seit Beginn des neuen Jahrtausends auf hohem Niveau stabilisiert. Der Trend entspricht weitgehend jenem der übrigen Nachfolgestaaten der Sowjetunion und speziell der zentralasiatischen Staaten ( $\bullet$ Abb. 1).

Als unabhängige Risikofaktoren für die Entwicklung einer pulmonalen Tuberkulose in Russland wurden beschrieben: Armut und Arbeitslosigkeit, Drogenkonsum, Diabetes, vorheriger Aufenthalt im Gefängnis oder in Untersuchungshaft, Tuberkulose eines Verwandten im Haushalt, das Trinken unpasteurisierter Milch sowie geringer Wohn- und Lebensraum (pro Person) [3]. Ein erhöhtes Tuberkuloserisiko findet sich zudem bei Ärzten und medizinischem Personal in russischen Tuberkuloseeinrichtungen und -Laboren $[4,5]$.

Als Risikofaktoren für den Tod an Tuberkulose in Russland (während einer Behandlung) wurden beschrieben: Obdachlosigkeit, Arbeitslosigkeit, fortgeschrittene Erkrankung zum Zeitpunkt der 


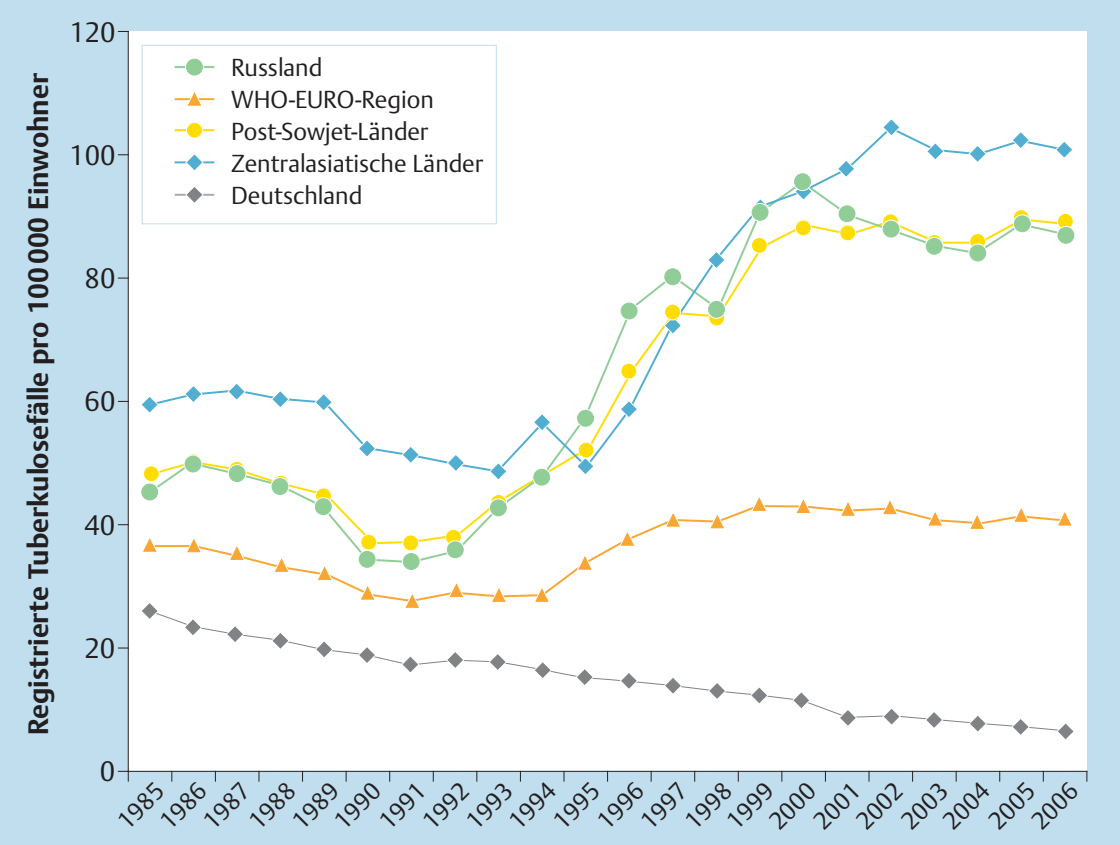

Abb. 1 Tuberkuloseraten in Russland im Vergleich mit anderen Nachfolgestaaten der Sowjetunion, der WHO-Euro-Region insgesamt sowie Deutschland (1985-2006).

Diagnose, Drogenkonsum sowie diverse Begleiterkrankungen $[6,7]$.

Sehr hoch ist die Zahl der Tuberkulosepatienten in Haftanstalten. Zum Ende des Jahres 2006 waren ca. 47000 Tuberkulosepatienten in russischen Gefängnissen bzw. Untersuchungshaft gemeldet (prävalente Fälle) [8]. Nach fast vierfachem Anstieg in den 90er-Jahren ist die Melderate jedoch seit einigen Jahren rückläufig [9]. 2006 war sie mit 1285 neuen Fällen pro 100000 Gefangene noch mehr als 18-mal so hoch wie im zivilen Bereich. Als Risikofaktoren für Tuberkulose in Gefängnissen wurden u.a. Überfüllung (Gefangene pro Zelle) und illegaler Drogenkonsum beschrieben [10].

\section{DOTS und Behandlungsergebnisse in Russland}

Mitte der 90er-Jahre wurden in verschiedenen Regionen Russlands Pilotprojekte gestartet, um das von der WHO entwickelte Modell der Tuberkulosebehandlung und -kontrolle, „DOTS“, einzuführen [11 - 13]. Dabei konnte gezeigt werden, dass die internationale Strategie im Vergleich zum russischen Kontrollmodell effektiv [14] und deutlich kosteneffizienter [15] ist. Jedoch wurden hohe MDR-Raten [13], Stigmatisierung [16,17] und niedrige Behandlungscompliance, speziell bei sozial schwachen $[18,19]$ bzw. alkohol- und drogenabhängigen Patienten [20,21], als Barrieren für eine erfolgreiche Behandlung identifiziert. Erst seit der offiziellen Anerkennung der WHO-Behandlungsstandards durch Russland im Jahr 2003 [22] gibt es Bestrebungen, diese auch in den übrigen der 79 Verwaltungsregionen durchzusetzen. Zwar stieg dadurch der Anteil der Bevölkerung, welcher Zugang zur Tuberkulosebehandlung nach der DOTS-Strategie hat, im Zeitraum 2001 bis 2006 offiziell von 16\% auf 84\% [8]. Jedoch bleiben die Behandlungsergebnisse unter den Erwartungen: 2005 wurde landesweit eine erfolgreiche Behandlung in nur 58\% (neue, sputumpositive Fälle) bzw. 31\% (Rezidivfälle) erreicht [23].

\section{Charakteristika und Besonderheiten der Tuberkulose-} kontrolle in Russland

Tuberkulosespezifisches Kontroll- und Versorgungssystem Kontrolle, Diagnostik und Behandlung der Tuberkulose sind in Russland im Rahmen eines zentralisierten, krankheitsspezifischen Systems organisiert - weitgehend unabhängig vom allgemeinen öffentlichen Gesundheitssystem. Zentrale Einrichtungen dieses Systems sind die regionalen Tuberkulose-Dispensaires, in denen die Bevölkerung bei Tuberkuloseverdacht ambulant untersucht und bestätigte Fälle registriert werden. An die Dispensaires angebunden ist ein ausgedehntes Netzwerk aus spezialisierten Tuberkulosekrankenhäusern und Sanatorien mit derzeit rund 78000 Betten. Die Krankenversorgung geschieht durch ca. 40000 medizinische Angestellte mit zumeist krankheitsspezifischer Ausbildung, darunter ca. 8500 Ärzte, so genannte „Phthisiater“ [8].

\section{Bevölkerungsscreening für Tuberkulose}

Entgegen internationaler Praxis hält Russland an ausgedehnten Bevölkerungsscreenings auf Tuberkulose mittels Röntgen (Fluorographie) und Mantoux-Hauttests fest [24].

Laut offiziellen Angaben wurden 2006 rund 61 Millionen Menschen mit Röntgen-Fluorographie auf Tuberkulose untersucht (43\% der Bevölkerung) [25]. Der Anteil der Tuberkulosefälle, die durch Screenings identifiziert wurden, liegt jedes Jahr bei über $50 \%$ aller neuen Fälle $[8,25]$.

Die Praxis von Massenuntersuchungen bei Erwachsenen beruht auf der Vorstellung, dass die Tuberkulose in einem nicht-infektiösen Frühstadium identifiziert und dann effektiver behandelt werden kann [26]. Zudem beruft sich Russland auf das Recht der Bevölkerung auf ein reduziertes Infektionsrisiko [27,28].

Kinder bis zum 15. Lebensjahr werden laut Vorschrift einmal pro Jahr per Hauttest nach Mantoux sowie zusätzlich bei Tuberkulosekontakt getestet $[26,27]$. Russland hält auch an der BCG-Impfung bzw. an der Revakzination bei negativem Mantoux-Test fest. Modernere Testverfahren wie Interferon-gamma-basierte Tests werden in Russland nicht angewendet. Das Screening ab dem 15. Lebensjahr wird mittels Röntgen-Fluorographie der Lunge 


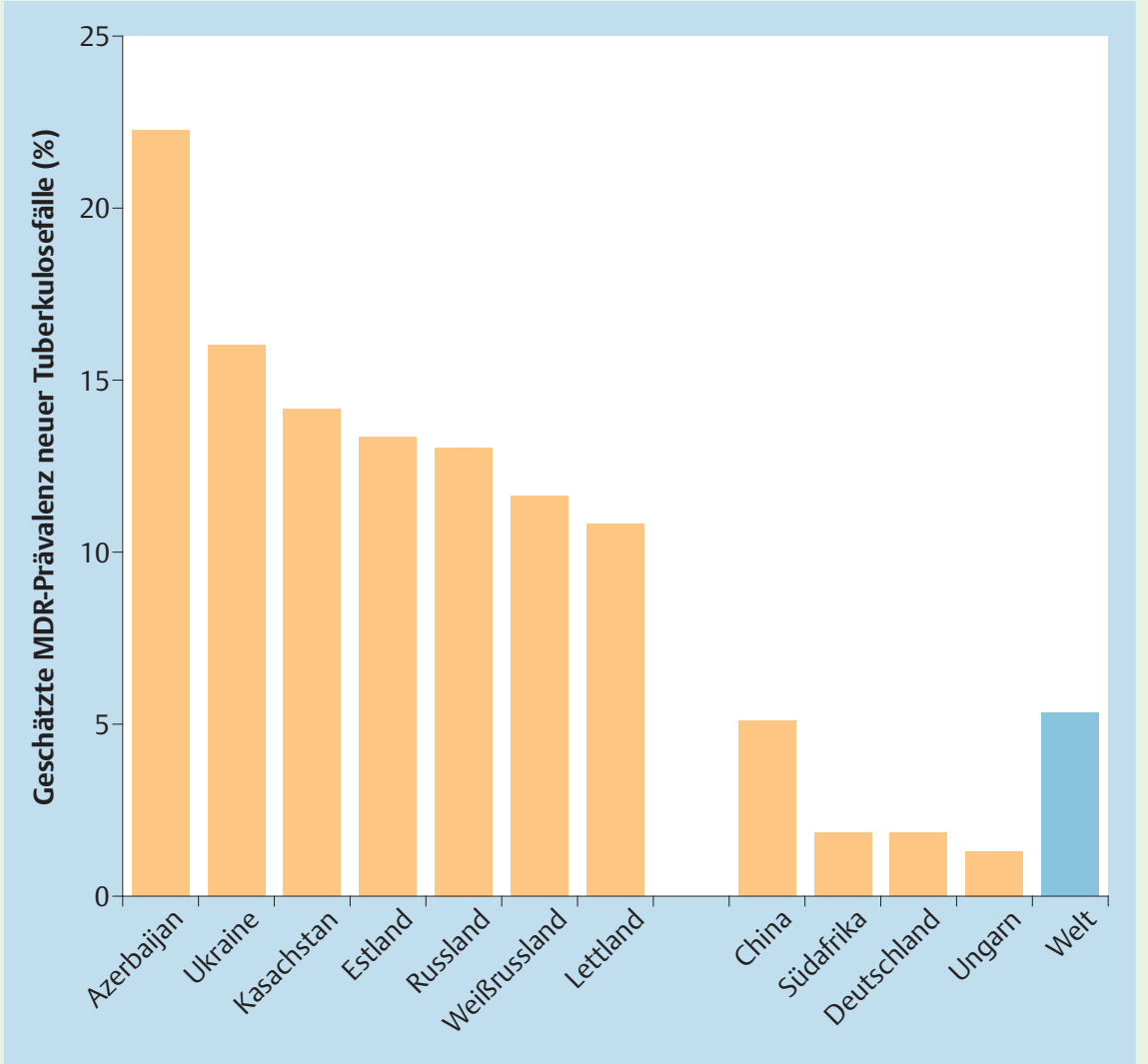

Abb. 2 Geschätzte MDR-Prävalenz (\% neue, nicht zuvor behandelte Fälle) ausgewählter Länder der ehemaligen Sowjetunion im Vergleich mit China, Südafrika, Deutschland und Ungarn (Quelle: WHO). mindestens einmal alle zwei Jahre durchgeführt. Bestimmte Risikogruppen (Mitarbeiter von Krankeneinrichtungen und Sozialdiensten, HIV-Positive, Obdachlose und Migranten) werden einbis zweimal pro Jahr getestet. Weiterhin existieren Vorschriften für außerordentliche Screenings [26,27]. Personen die zum Zeitpunkt des Screenings Symptome vorweisen, werden zusätzlich bakteriologisch untersucht [29].

Russland plant gegenwärtig die Ausweitung der Bevölkerungsscreenings in den Regionen; neue digitale Röntgeneinheiten werden derzeit zur Verfügung gestellt $[24,30]$.

\section{Diagnosestellung und Klassifizierung basieren im Schwerpunkt eher auf Röntgenbefunden als auf mikrobiologischer Diagnostik}

Neben den Screeninguntersuchungen haben Röntgenbefunde in Russland traditionell eine entscheidende Bedeutung bei der Diagnostik und der klinisch-epidemiologischen Klassifizierung der Tuberkulose. Das russische Klassifikationssystem basiert überwiegend auf klinisch-radiologischen Kriterien wie der Lokalisation, Ausbreitung, den pathologischen Eigenschaften von Läsionen, Krankheitsphasen, Komplikationen und residualen Veränderungen der betroffenen Organe [31]. Zwar war der kulturelle Erregernachweis traditionell überwiegend Bestandteil der Tuberkulosediagnostik. Ein flächendeckendes und qualitätsgesichertes Labornetzwerk existiert jedoch nicht. Das Kriterium der „bakteriellen Exkretion“, also ob und wie lange ein Patient als bakteriell positiv gilt, unterscheidet sich deutlich von internationalen Standards. So bleiben u.a. Patienten mit initial positivem Erregernachweis bis zu 3 Jahre nach dem ersten negativen Test als „bakterielle Ausscheider“ registriert. Rezidivfälle gingen traditionell nicht in die Berechnung der Inzidenz mit ein [32].

\section{„Individualisierte“ vs. standardisierte Behandlung in stationären Einrichtungen}

Im Gegensatz zur standardisierten Therapie nach internationalem Modell favorisiert das russische Kontrollmodell eine „individualisierte“ Tuberkulosebehandlung: Nach Diagnosestellung erhält der Patient eine Kombination aus Antibiotika und sogenannten Immunmodulatoren, die hinsichtlich Dosis, Therapiedauer und Applikation (oral, i.v., intrabronchial) vom zu behandelnden Arzt anzupassen ist. Darüber hinaus werden häufig nicht-medikamentöse Therapieansätze verfolgt, die von Galvanisierung über Vibromassage bis hin zur Retransfusion von UV-bestrahlten Leukozyten reichen [14]. Etwa 5-10\% aller russischen Tuberkulosepatienten werden chirurgisch behandelt [8].

Die Behandlung geschieht traditionell im stationären Bereich und dauert im Schnitt deutlich länger. Wiederholte Einweisungen und multiple Nachuntersuchungen sind üblich [33-35].

\section{Hohe Prävalenz multiresistenter Erreger}

Russland und die übrigen Staaten der ehemaligen Sowjetunion verzeichnen die weltweit höchsten Raten multiresistenter Tuberkulose. ${ }^{1}$

In den letzten Jahren wurden in einzelnen Regionen Russlands variabel hohe MDR-Raten zwischen 3 und 23\% der neuen Fälle [13,36-40] bzw. bis zu 55\% der zuvor behandelten Fälle [38] festgestellt. Besonders hoch ist der MDR-Anteil in Gefängnissen [41-43].

Bisher liegen jedoch landesweit nur unzureichende Daten für eine genaue epidemiologische Erhebung vor. Lediglich $24 \%$ der Tuberkulosefälle wurden 2006 auf Resistenzen getestet

\footnotetext{
1 Per definitionem wird dann von einem multiresistenten Erreger gesprochen, wenn in vitro Resistenzen mindestens gegen die zwei Erstlinienmedikamente Isoniazid und Rifampicin nachgewiesen werden.
} 
( Tab. 1). Die WHO schätzt die Prävalenz der MDR-Tuberkulose in Russland für das Jahr 2006 unabhängig vom Behandlungsstatus auf 19,4\% (95\% CI: 17,1 - 24,6\%) [39]. Vergleichbare MDR-Prävalenzraten finden sich auch in den übrigen Nachfolgestaaten der ehemaligen Sowjetunion ( $\bullet$ Abb. 2).

Darüber hinaus liegen in jüngster Zeit erste Berichte über XDRTuberkulosefälle $^{2}$ in Russland vor. In der sibirischen Region Tomsk litten im Zeitraum 2000-2004 insgesamt 29 von 608 MDR-Tuberkulosefällen an dieser noch schwieriger zu behandelnden Form der Erkrankung [44].

Als Risikofaktoren einer multiresistenten Tuberkulose in Russland wurden beschrieben: gegenwärtiger Gefängnisaufenthalt [13,37,38], Infektionen mit dem Beijing-Stamm [45-47], Alkoholmissbrauch [48], Hospitalisierung zu Beginn oder während der Behandlung [49] sowie Rückfall und Therapieversagen [13]. Zudem wurde ein Zusammenhang zwischen sozialer Armut als Barriere einer erfolgreichen Behandlung und der Entstehung von Multiresistenzen diskutiert [50].

\section{Ineffiziente Finanzierungssysteme für}

Tuberkulosebehandlung und -kontrolle

Tuberkulosekontrolle in Russland ist um ein Vielfaches teurer als in anderen High Burden Countries. Ursachen hierfür sind die Instandhaltung des Krankenhausnetzwerks, hohe Personalkosten [51] sowie die Praxis langfristiger stationärer Behandlungen mit multiplen Nachuntersuchungen, selbst von initial sputumnegativen Patienten [33].

Das Ausmaß der stationären Tuberkulosebehandlung (Verweildauer, Einweisungen) in den Regionen richtet sich dabei eher nach der Bettenkapazität als nach dem tatsächlichen medizinischen Bedarf [52].

Grund hierfür sind simple Per-Capita- und Fee-for-Service-Finanzierungssysteme, die Anreize für die Krankenhäuser schaffen, Betten zu füllen und Patienten langfristig zu behandeln bzw. wiederholt einzuweisen [53]. Dabei richten sich Einweisungen und Behandlungsdauer auch nach sozialen Maßstäben, da Tuberkulosekrankenhäuser besonders in den kalten Monaten das Fehlen von sozialen Sicherungssystemen kompensieren [54,55]. Trotz deutlicher Überkapazitäten [34] sind die Einrichtungen hinsichtlich der Bettenbelegung jedes Jahr nahezu vollständig ausgelastet [52].

Hohe Kosten entstehen weiterhin durch die Identifizierung von Patienten im Rahmen der Massenscreenings [51] sowie zunehmend durch die Behandlung multiresistenter Patienten.

\section{Diskussion}

Ein steiler Anstieg der Fall- und Sterbezahlen sowie hohe Multiresistenzraten in Gefängnissen und in der Zivilbevölkerung sind die Hauptkennzeichen einer Tuberkuloseepidemie, die sich in den 90er-Jahren auf dem Gebiet der ehemaligen Sowjetunion ausgebreitet hat. Obgleich die Meldedaten mit Vorsicht interpretiert werden müssen, wird ein tatsächlicher Anstieg der Tuberkuloseinzidenz in Russland nicht bezweifelt $[32,56]$.

\footnotetext{
${ }^{2}$ XDR- (extensively multidrug-resistant) Tuberkulose: Neben Isoniazid und Rifampicin (sowie weiteren Erstlinienmedikamenten) werden zusätzlich Resistenzen gegen ein Zweitlinienmedikament der Quinolonreihe sowie mindestens eines der Medikamente Kanamycin, Capreomycin oder Amikazin nachgewiesen.
}

Als Ursachen der Epidemie wurden der Zusammenbruch des sowjetischen Gesundheitssystems [57,58], inadäquate und unterfinanzierte Kontrollprogramme [59-61], Multiresistenzen und Medikamentenengpässe [62] sowie allgemein Wirtschaftskrisen und wachsende Armut in der Bevölkerung $[24,63,64]$ diskutiert.

Die Bilanz der Tuberkulosekontrolle fällt bisher schlecht aus. Trotz Ausweitung der internationalen Kontrollstrategie verfehlte Russland die weltweite Zielvorgabe der globalen Stop-TB-Strategie [65] hinsichtlich der Behandlungsergebnisse deutlich (2005:58\% vs. 85\% Heilung neuer sputumpositiver Patienten). Seit Anfang des neuen Jahrhunderts haben sich die Tuberkulosezahlen auf hohem Niveau stabilisiert; ein nennenswerter Rückgang ist bis zuletzt ausgeblieben. Auch die Zahl Tuberkulose-assoziierter Sterbefälle bleibt unverändert hoch.

Die Ergebnisse der DOTS-Strategie in Russland stehen im Kontrast zu den Erfahrungen aus anderen Ländern: In Bangladesh [66] und 5 weiteren High Burden Countries führte die Einführung von DOTS zu Behandlungserfolgen von über $90 \%$ der sputumpositiven Fälle [23]. In Peru kam es durch DOTS trotz verbesserter Identifizierung von Tuberkulosefällen zu einem deutlichen Rückgang der Fall- und Sterbezahlen [67]. Auch in China reduzierte die Einführung der standardisierten Therapie wesentlich die Sterblichkeit der Tuberkulose [68].

\section{Mykobakterielle Diagnostik und Kontrolle der MDR-Tuberkulose}

Wichtigste Ursache für die bislang schlechte Bilanz der Tuberkulosekontrolle in Russland ist der hohe Anteil multiresistenter Erkrankungen.

Die Praxis sogenannter individualisierter Tuberkulosebehandlungen ohne konsequenten Erregernachweis sowie Medikamentenengpässe [69] führten zur Entstehung von Medikamentenresistenzen und einer Generation von Patienten, die in Russland als „chronische Fälle“ bezeichnet werden, weil sie auf die üblichen Tuberkulosemedikamente nicht ansprechen. Diese waren Ausgangspunkt dafür, dass sich multiresistente Stämme in der Zivilgesellschaft Russlands ausbreiten konnten [70,71]. Kimerling et al. wiesen bereits im Jahr 2000 darauf hin, dass durch die primäre Übertragung multiresistenter Erreger in Russland ein zweiter Mechanismus der MDR-Epidemie entsteht, der unabhängig vom Prozess der Entstehung von Multiresistenzen wirkt und der somit einer eigenständigen Kontrollstrategie bedarf [28].

Die Russische Föderation erkennt inzwischen die Notwendigkeit des mikroskopischen und kulturellen Erregernachweises mit Resistenztest an [72,73]. Der Auf- und Ausbau eines landesweiten Labornetzwerks und die Ausbildung von Laborfachkräften sind derzeit Gegenstand des Föderalen Programms „Tuberkulose“ der Russischen Föderation. Finanziert wird das Programm mit Krediten der Weltbank sowie durch einen Grant im Rahmen der 4. Runde des Global Fund to Fight AIDS, Tuberculosis and Malaria (GFATM) [74].

Im Rahmen des Global-Fund-Projekts wurden in den vergangenen zwei Jahren insgesamt neun so genannte Exzellenzzentren für die Diagnostik und Behandlung der MDR-Tuberkulose im zivilen und im Gefängnisbereich etabliert. Resistenztests wurden landesweit in 92 Laboren eingeführt [75]. Die weitere Ausstattung von Laboren sowie der Ausbau der externen Qualitätskontrolle sind vorgesehen. 
Standardisierte Behandlung der Tuberkulose und der MDR-Tuberkulose

Die von der WHO empfohlene medikamentöse Kombinationstherapie ist nach Einführung einheitlicher Therapieprotokolle mittlerweile Grundlage der Tuberkulosebehandlung in den meisten Regionen. Jedoch existieren parallel auch weiterhin individualisierte Behandlungskonzepte (persönliche Kommunikation). Priorität hat derzeit die Ausweitung der Behandlung multiresistenter Tuberkulosefälle in Gefängnissen und zivilen Einrichtungen nach der DOTSplus-Strategie. Im Jahr 2006 hatten nur 4500 MDR-TB-Patienten Zugang zu einer Therapie mit Medikamenten der zweiten Reihe. Pläne, bis zu 24000 MDR-TB-Patienten unter DOTSplus zu behandeln, konnten zuletzt aufgrund mangelnder Finanzierung nicht realisiert werden [23].

\section{Welche Rolle spielen Bevölkerungsscreenings?}

Russland sieht in Reihenuntersuchungen traditionell eine wichtige Maßnahme im Kampf gegen die Tuberkulose. Jedoch ist es durch sie bisher offenbar nicht gelungen, die Zahl der Übertragungen in der Bevölkerung zu reduzieren und damit die Tuberkuloseraten zu senken. Stattdessen ergeben sich erhebliche Probleme für die Tuberkulosekontrolle: Die Reihenuntersuchungen generieren in Russland offenbar eine hohe Zahl sputumnegativer Patienten ${ }^{3}$, bei denen die Diagnose weder mikrobiologisch bestätigt noch Resistenztests durchgeführt und ein Behandlungserfolg verlässlich dokumentiert werden kann. Hohe Kosten und die hohe Strahlenbelastung der Bevölkerung stehen zudem in keinem Verhältnis zum Nutzen einer möglicherweise erhöhten Sensitivität der Erfassung [76,77].

Die WHO lehnt aktive Reihenuntersuchungen mittels Röntgenuntersuchungen ab und favorisiert im Rahmen von DOTS die passive Identifizierung von Fällen bei einer entsprechenden Optimierung des Zugangs zur Gesundheitsversorgung [78].

Allerdings ist die Funktion der Screenings für die Identifizierung von infektiösen Tuberkulosefällen (Case Finding) in Russland bisher wenig untersucht. Angesichts hoher (MDR-)Fallzahlen könnte eine umsichtige Anwendung von Reihenuntersuchungen in Risikopopulationen durchaus wirkungsvoll sein. Epidemiologische Studien sind notwendig, um den Einfluss von Bevölkerungsuntersuchungen auf die Kontrolle der Tuberkulose in Russland zu untersuchen.

\section{Tuberkulose und HIV|AIDS}

Von erheblicher Bedeutung für die Tuberkulosekontrolle ist die gegenwärtige HIV/AIDS-Epidemie in Russland. Laut Schätzungen von UNAIDS stieg die Zahl der HIV-Infizierten im Zeitraum 2001 - 2007 von 390000 auf 940000 . Im Jahr 2006 waren 2,3\% der getesteten Tuberkulosefälle HIV-positiv [23].

Die HIV-Prävalenz in der Gesamtbevölkerung (laut UNAIDS derzeit ca. 1,1\% der Erwachsenen) ist derzeit noch vergleichsweise moderat. Jedoch überschneiden sich in Russland die Populationen der Tuberkulosefälle und der Personen mit erhöhtem HIVRisiko deutlich $[79,80]$. Aus diesem Grund ist eine weitere Ausbreitung der (MDR-)Tuberkulose in Russland auf der Grundlage vermehrter HIV-Infektionen prinzipiell durchaus denkbar.

Vor allem russische Gefängnisse gelten als Ausgangspunkte einer kombinierten HIV/Tuberkulose-Epidemie [81]. Der Anteil HIVInfizierter Tuberkulosepatienten stieg dort in den vergangenen Jahren deutlich (2006:6\% aller Tuberkulosefälle) [8].

\footnotetext{
${ }^{3}$ Daten nicht publiziert, aber auf Anfrage erhältlich.
}

Die erfolgreiche Kontrolle der Tuberkulose in Russland ist somit abhängig von integrierten HIV/Tuberkulose-Programmen [82] und von der Überwachung und Kontrolle der HIV-Epidemie im Land. Priorität haben derzeit Programme zur Prävention von HIV in Risikopopulationen, die Beratung und Testung v.a. von Tuberkulosepatienten sowie die Einführung von Leitlinien zur Behandlung bei kombinierter Infektion/Erkrankung $[23,83]$.

\section{Reformbedarf der Finanzierungssysteme}

Hohe Personalkosten, die Praxis langfristiger stationärer Behandlungen sowie starre und ineffiziente Finanzierungssysteme führen in Russland zu einer Explosion der Kosten für Tuberkulosekontrolle. Gegenwärtig existiert eine Finanzierungslücke des Kontrollprogramms von ca. 153 Millionen US-Dollar (2008), davon fehlen 112 Millionen US-Dollar allein für die Behandlung von Patienten mit multiresistenter Tuberkulose.

Obgleich gezeigt werden konnte, dass DOTS in Russland deutlich Kosten einsparen kann [15], ist es unter der Strategie bisher weder zu einer Reform der Finanzierungssysteme noch zu einer Reduzierung der stationären Bettenkapazität und Behandlungsdauer gekommen [52].

Aufgrund der hohen Kosten ist eine Reform der Gesundheits-, Sozial- und Finanzierungssysteme, speziell aber die Reduzierung stationärer Bettenkapazitäten und der Ausbau ambulanter Behandlungsmöglichkeiten notwendig.

Unsere Studie ist limitiert durch die Beschränkung auf die englischsprachige Literatur. Eine systematische Analyse und Auswertung der russischsprachigen Fachliteratur war aus Gründen der Finanzierung und Machbarkeit leider nicht möglich. Ein Gewinn weiterer wichtiger Aspekte und Informationen durch die systematische Auswertung der russischen Fachliteratur kann prinzipiell nicht ausgeschlossen werden. Allerdings gehen wir von einer objektiven Abbildung der Situation in Russland in den untersuchten englischsprachigen Artikeln aus, zumal eine große Zahl russischer Tuberkulosespezialisten und Wissenschaftler an den Artikeln direkt beteiligt war. Die überwiegende Mehrheit der nichtrussischen Autoren war vor Ort in Russland im Rahmen von bi- und multilateralen Kontrollprojekten tätig.

\section{Fazit}

Gegenwärtig zählen der Auf- und Ausbau einer qualitätsgesicherten mikrobiologischen Diagnostik und Resistenztestung, die Prävention, Kontrolle und Behandlung der MDR-Tuberkulose sowie Prävention und Management von Tuberkulose/HIV-Koinfektionen zu den Prioritäten der Tuberkulosekontrolle in Russland. Darüber hinaus entscheidend ist die Reform der Gesundheits- und Finanzierungssysteme.

Durch die von der Russischen Föderation mithilfe der WHO und internationaler Partner etablierten Programme konnten erste Teilerfolge erzielt werden. Dennoch bleibt die Lage besonders durch die Zunahme multiresistenter Tuberkulosefälle angespannt.

Die Tuberkulosesituation in der Europäischen Union ist dabei unmittelbar mit jener der Sowjet-Nachfolgestaaten verbunden - besonders vor dem Hintergrund steigender Medikamentenresistenzen. In Deutschland geben jährlich ein Fünftel bis ein Viertel aller registrierten Tuberkulosefälle an, in einem Land der ehemaligen Sowjetunion geboren zu sein [84]. Von den 184 multiresistenten Tuberkulosefällen, die 2004-2006 in Deutschland diag- 
nostiziert wurden, stammten 148 (80\%) aus den ehemaligen Sowjetländern ([85], vgl. auch $\bullet$ Tab. 1).

Für eine erfolgreiche Tuberkulosekontrolle in Russland und in den übrigen Staaten der ehemaligen Sowjetunion spielen auch deshalb internationale Kooperationen in den kommenden Jahren eine wichtige Rolle. Den Rahmen dafür kann die Stop TB Partnership bilden, eine Initiative von Regierungen und Nichtregierungsorganisationen, internationalen Organisationen und des privaten Sektors.

\section{Finanzielle Unterstützung}

Diese Arbeit wurde durch eine Forschungsförderung des Bundesministeriums für Bildung und Forschung (BMBF; RUS 05/A23) sowie durch die Günther-Labes-Stiftung unterstützt.

\section{Literatur}

1 World Health Organization. Pursue high-quality DOTS expansion and enhancement. WHO. http://www.who.int/tb/dots/en/index.html

2 Estimated epidemiological burden of TB 2006, Table 1.2, Global tuberculosis control, Surveillance, Planning, Financing. WHO Report 2008. Geneva, Switzerland: World Health Organization, 2008: 19

3 Coker $R$ et al. Risk factors for pulmonary tuberculosis in Russia: Casecontrol study. British Medical Journal 2006; 332: 85 - 87

4 Dimitrova $B$ et al. Increased risk of tuberculosis among health care workers in Samara Oblast, Russia: Analysis of notification data. International Journal of Tuberculosis and Lung Disease 2005; 9: 43-48

5 Drobniewski $F$ et al. Rates of latent tuberculosis in health care staff in Russia. PLoS Medicine 2007; 4: 0273 - 0279

6 Dewan PK et al. Risk factors for death during tuberculosis treatment in Orel, Russia. International Journal of Tuberculosis and Lung Disease 2004; 8: 598 - 602

7 Kourbatova EV et al. Risk factors for mortality among adult patients with newly diagnosed tuberculosis in Samara, Russia. International Journal of Tuberculosis and Lung Disease 2006; 10: 1224-1230

8 Tuberculosis in the Russian Federation 2006. Analytical Review. (Russian). Moscow: Russian Ministry of Health and Social Development, 2007

9 Bobrik A et al. Prison health in Russia: the larger picture.[see comment]. Journal of Public Health Policy 2005; 26: 30 - 59

10 Lobacheva T, Asikainen T, Giesecke J. Risk factors for developing tuberculosis in remand prisons in St. Petersburg, Russia - A case-control study. European Journal of Epidemiology 2007; 22: 121 - 127

11 Kherosheva T et al. Encouraging outcomes in the first year of a TB control demonstration program: Orel Oblast, Russia. Int J Tuberc Lung Dis 2003; 7: 1045 - 1051

12 Ruohonen RP et al. Implementation of the DOTS strategy for tuberculosis in the Leningrad Region, Russian Federation (1998-1999). International Journal of Tuberculosis and Lung Disease 2002; 6: 192-197

13 Balabanova $Y$ et al. The Directly Observed Therapy Short-Course (DOTS) strategy in Samara Oblast, Russian Federation. Respiratory Research 2006; 7: 44

14 Mawer $C$ et al. Comparison of the effectiveness of WHO short-course chemotherapy and standard Russian antituberculous regimens in Tomsk, western Siberia. Lancet 2001; 358: 445-449

15 Migliori GB et al. Cost-effectiveness analysis of tuberculosis control policies in Ivanovo Oblast, Russian federation. Bulletin of the World Health Organization 1998; 76: 475-483

16 Woith WM, Larson JL. Delay in seeking treatment and adherence to tuberculosis medications in Russia: a survey of patients from two clinics. International Journal of Nursing Studies 2008; 45: 1163 - 1174

17 Dimitrova $B$ et al. Health service providers" perceptions of barriers to tuberculosis care in Russia. Health Policy and Planning 2006; 21: 265 274

18 Jakubowiak WM et al. Risk factors associated with default among new pulmonary TB patients and social support in six Russian regions. International Journal of Tuberculosis and Lung Disease 2007; 11: 46-53

19 Jakubowiak WM et al. Treatment default among new smear-positive pulmonary TB patients in Russian regions [2]. International Journal of Tuberculosis and Lung Disease 2007; 11: 353 - 354
20 Gelmanova IY et al. Barriers to successful tuberculosis treatment in Tomsk, Russian Federation: non-adherence, default and the acquisition of multidrug resistance. Bull World Health Organ 2007; 85: 703 711

21 Fry RS et al. Barriers to completion of tuberculosis treatment among prisoners and former prisoners in St Petersburg, Russia. International Journal of Tuberculosis and Lung Disease 2005; 9: 1027-1033

22 Prikas RF. No. 109. Moscow: The Ministry of Health and Social Welfare of the Russian Federation, 2003.

23 World Health Organization. Global tuberculosis control (Surveillance, Planning, Financing); Country Profile: Russian Federation. WHO Report 2008. Geneva, Switzerland: World Health Organization, 2008: $141-144$

24 Perelman MI. Tuberculosis in Russia. International Journal of Tuberculosis and Lung Disease 2000; 4: 1097-1103

25 Skachkova EI, Dergachiev AV. [Organisation der Identifizierung und Erstuntersuchung von Tuberkulosepatienten in der Russischen Föderation], Institute of Public Health Organization and Informatics, Ministry of Health and Social Welfare of the Russian Federation. Russisch. 2007.

26 [Screening-Methoden zur Identifizierung von Tuberkulosepatienten. Analytischer Bericht], Institute of Public Health Organization and Informatics, Ministry of Health and Social Welfare of the Russian Federation. Russisch. 15.10.2007. 2007.

27 Organization of differentiated fluorographic examinations of the population for detection of thoracic diseases. Guidelines No.95/42. Ministry of Health and Medical Industry of the Russian Federation; State Sanitary and Epidemiological Surveillance Committee of the Russian Federation, 21./22.02.1996.

28 Kimerling ME. The Russian equation: An evolving paradigm in tuberculosis control. International Journal of Tuberculosis and Lung Disease 2000; 4 (12 Suppl. 2): S160-S167

29 Resolution Nr. 892 der Regierung der Russischen Föderation, 25.12.2001.

30 Heifets L, Iseman MD. Leo Tolstoy, TB in Russia, and American perceptions. International Journal of Tuberculosis and Lung Disease 2002; 6 : 939-941

31 Prikas RF. No. 324. [Clinical classification of Tuberculosis], Moscow: The Ministry of Health and Social Welfare of the Russian Federation. (Russian). 1995

32 Coker RJ et al. Tuberculosis control in Samara Oblast, Russia: institutional and regulatory environment. International Journal of Tuberculosis \& Lung Disease 2003; 7: 920-932

33 Atun RA et al. Costs and outcomes of tuberculosis control in the Russian Federation: retrospective cohort analysis. Health Policy \& Planning 2006; $21: 353-364$

34 Floyd $K$ et al. Health-systems efficiency in the Russian Federation: Tuberculosis control. Bulletin of the World Health Organization 2006; 84: $43-51$

35 Atun RA et al. Implementing WHO DOTS strategy in the Russian Federation: Stakeholder attitudes. Health Policy 2005; 74: 122 - 132

36 Kherosheva T et al. Encouraging outcomes in the first year of a TB control demonstration program: Orel Oblast, Russia. International Journal of Tuberculosis and Lung Disease 2003; 7: 1045-1051

37 Spradling $P$ et al. Anti-tuberculosis drug resistance in community and prison patients, Orel Oblast, Russian Federation. International Journal of Tuberculosis and Lung Disease 2002; 6: 757 - 762

38 Ruddy $M$ et al. Rates of drug resistance and risk factor analysis in civilian and prison patients with tuberculosis in Samara Region, Russia. Thorax 2005; 60: 130-135

39 Anti-tuberculosis Drug Resistance in the World, 4th Global Report 2008; The WHO/IUATLD Global Project on Tuberculosis Drug Resistance Surveillance 2002-2007. http://www.who.int/tb/publications/ 2008/drs_report4_26feb08.pdf. 2008

40 Migliori GB et al. Frequency of recurrence among MDR-tB cases "successfully" treated with standardised short-course chemotherapy. International Journal of Tuberculosis \& Lung Disease 2002; 6: 858-864

41 Portaels F, Rigouts L, Bastian I. Addressing multidrug-resistant tuberculosis in penitentiary hospitals and in the general population of the former Soviet Union. International Journal of Tuberculosis and Lung Disease 1999; 3: $582-588$

42 Kimerling $M E$ et al. Inadequacy of the current WHO re-treatment regimen in a central Siberian prison: Treatment failure and MDR-TB. International Journal of Tuberculosis and Lung Disease 1999; 3: 451 453 
43 Bonnet $M$ et al. Does one size fit all? Drug resistance and standard treatments: Results of six tuberculosis programmes in former Soviet countries. International Journal of Tuberculosis and Lung Disease 2005; 9: 1147 - 1154

44 Keshavjee $S$ et al. Treatment of extensively drug-resistant tuberculosis in Tomsk, Russia: a retrospective cohort study. Lancet 2008; 372: $1403-1409$

45 Drobniewski F et al. Drug-resistant tuberculosis, clinical virulence, and the dominance of the Beijing strain family in Russia.[see comment]. JAMA 2005; 293: 2726-2731

46 Ignatova A et al. Predominance of multi-drug-resistant LAM and Beijing family strains among Mycobacterium tuberculosis isolates recovered from prison inmates in Tula Region, Russia. Journal of Medical Microbiology 2006; 55: 1413-1418

47 Toungoussova OS, Bjune G, Caugant DA. Epidemic of tuberculosis in the former Soviet Union: social and biological reasons. Tuberculosis 2006; 86: $1-10$

48 Fleming MF et al. Alcohol and drug use disorders, HIV status and drug resistance in a sample of Russian TB patients. International Journal of Tuberculosis \& Lung Disease 2006; 10: 565 - 570

49 Gelmanova IY et al. Barriers to successful tuberculosis treatment in Tomsk, Russian Federation: Non-adherence, default and the acquisition of multidrug resistance. Bulletin of the World Health Organization 2007; 85: $703-711$

50 Keshavjee $S$ et al. Treating multidrug-resistant tuberculosis in Tomsk, Russia: Developing programs that address the linkage between poverty and disease. Reducing the Impact of Poverty on Health and Human Development: Scientific Approaches. Annals of the New York Academy of Sciences 2008; 1136: 1 - 11

51 WHO. World Health Organization: Workshop on „Improving the costeffectiveness of TB control in the Russian Federation: the role of inpatient care“. 2005; http://whqlibdoc.who.int/hq/2005/WHO_HTM_ TB_2005.357_2_eng.pdf

52 Marx FM et al. Reform of tuberculosis control and DOTS within Russian public health systems: an ecological study. Eur J Public Health 2007; 17: $98-103$

53 Atun RA et al. Barriers to sustainable tuberculosis control in the Russian Federation health system. Bulletin of the World Health Organization 2005; 83: 217-223

54 Atun RA et al. Social factors influencing hospital utilisation by tuberculosis patients in the Russian Federation: analysis of routinely collected data. Int J Tuberc Lung Dis 2005; 9: 1140-1146

55 Atun $R$ et al. Seasonal variation and hospital utilization for tuberculosis in Russia: hospitals as social care institutions. The European Journal of Public Health 2005; 15: 350 - 354; doi:10.1093/eurpub/cki018 2005.

56 Farmer PE, Kim Jy. Resurgent TB in Russia: Do we know enough to act? European Journal of Public Health 2000; 10: 150-152

57 Shilova MV, Dye C. The resurgence of tuberculosis in Russia. Philos Trans R Soc Lond B Biol Sci 2001; 356: 1069-1075

58 WHO. Highlights on health in the Russian Federation, 2005. http:// www.euro.who.int/highlights 2006

59 Coker R, Atun R, McKee M. Untangling Gordian knots: Improving tuberculosis control through the development of "programme theories". International Journal of Health Planning and Management 2004; 19 : $217-226$

60 Coker RJ et al. Health system frailties in tuberculosis service provision in Russia: An analysis through the lens of formal nutritional support. Public Health 2005; 119: 837 - 843

61 Kipp W. Tuberculosis in the eastern European countries and the former USSR. Healthcare Management Forum 2001; 14: 54-59

62 Yerokhin VV, Punga VV, Rybka LN. Tuberculosis in Russia and the problem of multiple drug resistance. Annals of the New York Academy of Sciences 2001; 953: $133-137$
63 Rimashevskaia N. Poverty and Health in Russia. Sociological Research 2004; $43: 26$

64 Shilova MV. Specific features of the spread of tuberculosis in Russia at the end of the 20th century. Annals of the New York Academy of Sciences 2001; 953: 124-132

65 Achieving the targets: What needs to be done. The Global Plan to Stop TB 2006-2015. Stop-TB Partnership. http://www.stoptb.org/globalplan/assets/documents/GP_P1_S2.pdf

66 Mushtaque A, Chowdhury R. Success with the DOTS strategy. Lancet 1999; 353: 1003 - 1004

67 Suarez PG et al. The dynamics of tuberculosis in response to 10 years of intensive control effort in Peru. J Infect Dis 2001; 184: $473-478$

68 Dye $C$ et al. Evaluating the impact of tuberculosis control: number of deaths prevented by short-course chemotherapy in China. Int J Epidemiol 2000; 29: 558 - 564

69 Banatvala N, Peremitin GG. Tuberculosis, Russia, and the Holy Grail. Lancet 1999; 353: $999-1000$

70 Kimerling ME et al. The risk of MDR-TB and polyresistant tuberculosis among the civilian population of Tomsk city, Siberia, 1999. International Journal of Tuberculosis and Lung Disease 2003; 7: 866-872

71 Centers for Disease, C. and Prevention. Primary multidrug-resistant tuberculosis-Ivanovo Oblast, Russia, 1999. MMWR - Morbidity \& Mortality Weekly Report 1999; 48: 661 - 664

72 Prikas RF. No. 50. Moscow: The Ministry of Health and Social Welfare of the Russian Federation, 2004

73 Heifets $L$. WHO and Russia: The turning point in joint efforts against TB. International Journal of Tuberculosis and Lung Disease 2003; 7: 101 102

74 The Global Fund. Promoting a Strategic Response to TB Treatment and Care for Vulnerable Populations in the Russian Federation. Global Fund Grant No: RUS-405-G04-T. http://www.theglobalfund.org/programs/ grantdetails.aspx?CountryId=RUS\&compid=913\&grantid= 431\&lang=en

75 The Global Fund. Grant Performance Report. Russian Federation; Grant No: Rus-405-G04-T. 05.08.2008

76 Migliori GB et al. Cost-effectiveness analysis of tuberculosis control policies in Ivanovo Oblast, Russian Federation. Ivanovo Tuberculosis Project Study Group. Bull World Health Organ 1998; 76: 475-483

77 Golub JE et al. Active case finding of tuberculosis: historical perspective and future prospects. Int J Tuberc Lung Dis 2005; 9: 1183-1203

78 WHO, WHO Expert Committee on Tuberculosis. Nineth report. Geneva: World Health Organization, 1974 (WHO Technical Report Series, No. 552)

79 Kazionny B et al. Implications of the growing HIV-1 epidemic for tuberculosis control in Russia. Lancet 2001; 358: 1513 - 1514

80 Drobniewski FA et al. The "bear trap": The colliding epidemics of tuberculosis and HIV in Russia. International Journal of STD and AIDS 2004; 15: $641-646$

81 Drobniewski FA et al. Tuberculosis, HIV seroprevalence and intravenous drug abuse in prisoners. European Respiratory Journal 2005; 26 : $298-304$

82 Van Rie A et al. TB and HIV in St. Petersburg, Russia: A looming catastrophe? International Journal of Tuberculosis and Lung Disease 2005; 9: $740-745$

83 Webster P. World Bank approves loan to help Russia tackle HIV/AIDS and tuberculosis. Lancet 2003; 361: 1355

84 Bericht zur Epidemiologie der Tuberkulose in Deutschland für 2006. Robert Koch-Insitut, Berlin: 2008

85 Eker $B$ et al. Multidrug- and extensively drug-resistant tuberculosis, Germany. Emerg Infect Dis 2008; 14: 1700-1706 